\title{
Wnt Antagonist, Secreted Frizzled-Related Protein 4 (sFRP4), Increases Chemotherapeutic Response of Glioma Stem-Like Cells
}

\author{
Sudha Warrier,* Senthil Kumar Balu,* Alan Prem Kumar, $† \ddagger \S \llbracket$ Michael Millward,\# \\ and Arunasalam Dharmarajan\# ${ }^{1}$ \\ *Division of Cancer Stem Cells and Cardiovascular Regeneration, Manipal Institute of Regenerative Medicine, \\ Manipal University, Bangalore, India \\ $†$ Cancer Science Institute of Singapore, National University of Singapore, Singapore \\ \$Department of Pharmacology, Yong Loo Lin School of Medicine, National University of Singapore, Singapore \\ §Department of Biological Sciences, University of North Texas, Denton, TX, USA \\ IISchool of Biomedical Sciences, Faculty of Health Sciences, Curtin University, Western Australia, Australia \\ \#School of Anatomy, Physiology and Human Biology, The University of Western Australia, Perth, Western Australia, Australia
}

\begin{abstract}
Malignant gliomas have a highly tumorigenic subpopulation, termed cancer stem cells (CSCs), that drives tumor formation and proliferation. CSCs possess inherent resistance mechanisms against radiation- and chemotherapy-induced cancer cell death, enabling them to survive and initiate tumor recurrence. We examined the effect of secreted frizzled-related protein 4 (sFRP4), a Wnt signaling antagonist, in chemosensitizing the glioma cell line U138MG and glioma stem cells (GSCs) enriched from U138MG to chemotherapeutics. We found that SFRP4 alone and in combination with either doxorubicin or cisplatin induced apoptosis. Proliferation decreased substantially in GSC-enriched population as measured by MTT and BrdU assays. JC-1 and caspase-3 assays demonstrated that cell death was through the apoptotic pathway. sFRP4 treatment also decreased neurosphere formation and induced neuronal differentiation. Inhibition by sFRP4 was abolished by Wnt3a, indicating that sFRP4 acts through the frizzled receptor. Further indication that sFRP4 acts through the Wnt $\beta$-catenin pathway was provided by decrease in the $\beta$-catenin protein and decrease in the $\beta$-catenin-stimulated gene cyclin D1 upon sFRP4 induction. By real-time PCR, an increase in apoptotic markers Bax and p21, a decrease in pro-proliferative marker CycD1, and a decrease in the GSC marker CD133 were observed. These observations indicate that sFRP4 is able to sensitize glioma cells and stem cells to chemotherapeutics. We thus identified for the first time that SFRP4 could help to destroy cancer stem cells of glioma cell line, which would lead to effective treatment regimen to combat brain tumors.
\end{abstract}

Key words: Glioma stem cells (GSCs); Secreted frizzled-related protein 4 (sFRP4); Apoptosis; Chemosensitivity; Wnt antagonist

\section{INTRODUCTION}

Glioblastoma multiforme (GBM, grade IV of glioma) is one of the most aggressive and lethal forms of cancer having a median survival time of 15 months after diagnosis (1). The growth and progression of GBM depend on a highly tumorigenic, self-renewing population of stem/ initiating cells or glioma stem cells (GSCs) that contribute to tumor propagation and resistance to therapy. Cancer stem cells (CSCs) contribute to glioma radioresistance by an increase in DNA repair capacity through preferential activation of the DNA damage response checkpoints (2).

$\mathrm{Wnt} / \beta$-catenin signaling plays a role in the proliferation of glioma tumor cells and tumor progression.
Secreted frizzled-related protein 4 (sFRP4) is a member of the secreted frizzled-related protein family, which are extracellular inhibitors of Wnt that can antagonize both canonical and noncanonical Wnt pathways and has been implicated in different types of cancers $(3,4)$.

We have previously shown the presence of CSC-like cells from the human glioma cell line U138MG (5). In this study, we investigated the effect of this naturally occurring antagonist, sFRP4, on glioma cells and GSCs from the human glioma cell line U138MG. This study provides a new therapeutic strategy for targeting the stem cell population of gliomas using combinatorial drug treatment with sFRP4.

${ }^{1}$ Current address: School of Biomedical Sciences, Faculty of Health Sciences, Curtin University, Western Australia, Australia. Address correspondence to Sudha Warrier, Division of Cancer Stem Cells and Cardiovascular Regeneration, Manipal Institute of Regenerative Medicine, Manipal University, Bangalore 560 065, India. Tel: +91-80-28460671, 28460691;

Fax: +91-80 28460691; E-mail: sudha.warrier@ gmail.com or sudha.warrier@manipal.edu 


\section{MATERIALS AND METHODS}

\section{Cell Culture}

The U138MG grade 4 glioblastoma cell line was obtained from ATCC and cultured and maintained in DMEM/F-12 and DMEM-LG (Gibco) (1:1) containing 1× GlutaMax (Life Technologies), 10\% fetal bovine serum (Hyclone), and $100 \mathrm{U} / \mathrm{ml}$ PenStrep (Life Technologies). Serum-free medium (SFM) for sphere culture was carried out in basal medium (DMEM/F-12+DMEM-LG) with $100 \mathrm{U} / \mathrm{ml}$ PenStrep, $2 \mathrm{mM}$ GlutaMAX, and containing the growth factors bFGF (20 ng/ml), EGF (20 ng/ml) (both from R\&D Systems), and 1× B27 (GIBCO). All cells were cultured at $37^{\circ} \mathrm{C}$ in a humid incubator with $5 \% \mathrm{CO}_{2}$.

\section{Tumor Sphere Culture}

GSC-enriched populations of cells were obtained by plating a single cell suspension of U138MG cells at 5,000 cells $/ \mathrm{ml}$ in serum-free medium on nonadherent six-well plates (BD Biosciences) as reported by us previously (5). Briefly, cells were maintained in serum-free stem cell media (SFM) comprised of DMEM-F12 containing $20 \mathrm{ng} /$ $\mathrm{ml}$ each of epidermal growth factor (EGF), basic fibroblast growth factor (bFGF; R\&D Systems), leukemia inhibitory factor (LIF; Chemicon), B27 (1:50; Life Technologies), and $1 \%$ penicillin-streptomycin (Life Technologies). The medium was replenished once in 2 days by centrifuging for $3 \mathrm{~min}$ at $450 \times g$ at room temperature, aspirating the medium, and replacing it with fresh stem cell medium until the neurospheres were $>120 \mu \mathrm{m}$ in size as observed using an inverted phase contrast microscope (Nikon Eclipse TE 2000-S, Japan). Neurospheres were then characterized for the expression of GSC markers CD133, nestin, and ABCG2 protein using immunohistochemistry and qPCR. After identifying the enrichment of the spheres for GSC, they were used for further analysis.

\section{Chemotherapeutic Agent and Drug Treatment}

The drugs used in this study were purified sFRP4 (R\&D Systems), doxorubicin (Merck), and cisplatin (Sigma). Sensitization with sFRP4 was performed by adding sFRP4 to the cell culture at various concentrations for $12 \mathrm{~h}$. After sFRP4 treatment, drugs (doxorubicin or cisplatin at various concentrations) were added in fresh medium and incubated for $24 \mathrm{~h}$.

sFRP4 was tested at $0,10,50,75,100,125,150,200$, and $250 \mathrm{pg} / \mathrm{ml}$ for proliferation on both monolayer cells and GSC cells. The cells were chemosensitized with sFRP4 at the indicated concentrations for $12 \mathrm{~h}$. They were then washed and subjected to drugs, either doxorubicin or cisplatin. Doxorubicin was tested at 25,50 , and $100 \mathrm{ng} / \mathrm{ml}$. Cisplatin was tested at its $\mathrm{IC}_{50}$ value of $5 \mu \mathrm{M}$. Wnt3a was added at $20 \mathrm{ng} / \mathrm{ml}$. The drug treatment for the downstream analysis was optimized at $125 \mathrm{pg}$ of sFRP4 for $12 \mathrm{~h}$ followed by $50 \mathrm{ng} / \mathrm{ml}$ of doxorubicin for $24 \mathrm{~h}$. The MTT assay was used for the analysis of cellular proliferation.

\section{MTT Assay}

The TACS MTT assay kit (R\&D Systems) was used according to the manufacturer's protocol to measure cell metabolic viability.

Spheres. Spheres cultured in nonadherent SFM conditions in six-well plates were transferred to a non-tissue culture-treated flat-bottomed 96-well plate. After drug treatment, MTT assay (Merck) was performed as per the manufacturer's instructions. Plates were read at $595 \mathrm{~nm}$ using a Victor 3 Multilabel Plate Reader (Perkin-Elmer).

Monolayers. Ten thousand cells per well were plated in a flat-bottomed 96-well plate. After $12 \mathrm{~h}$, they were given a medium change, and the protein/drug was added. After drug treatment, MTT assay (Merck) was performed as per the manufacturer's instructions.

\section{BrdU Assay}

Monolayer cells were treated with sFRP4 followed by dox/cis as indicated previously. Cell proliferation was measured using the BrdU Cell Proliferation Assay Kit (Cell Signalling Tech Inc.) according to the manufacturer's protocol. Plates were read at $450 \mathrm{~nm}$ using Victor 3 Multilabel Plate Reader.

\section{Caspase-3 Assay}

GSCs were sensitized to sFRP4 followed by doxorubicin treatment as indicated previously. The activity of apoptosis-inducing caspase-3 was assayed using the EnzChek DEVD-CHO Caspase-3 Assay Kit (Invitrogen), which provides a fluorescent substrate R110. Intensity of the fluorescence is correlated with an increase in caspase- 3 activity, and the fluorescence intensity was measured using a Victor 3 Multilabel Plate Reader at 485/535 nm (Ex/Em).

\section{JC-1 Assay}

GSCs were treated with sFRP4 followed by doxorubicin treatment as indicated previously. The MitoProbe ${ }^{\mathrm{TM}} \mathrm{JC}-1$ Assay Kit (Invitrogen) was used to quantify mitochondrial membrane depolarization according to manufacturer's protocol. After the drug treatment, fluorescence at $590 \mathrm{~nm}$ (nonapoptotic cells) and $520 \mathrm{~nm}$ (apoptotic cells) was measured, and a ratio of 590:520 was calculated.

\section{Western Blot}

Protein expression levels of $\beta$-catenin, the downstream and central component of Wnt signaling, were determined by Western blotting. After drug treatment, total protein in each condition was isolated in RIPA buffer (Invitrogen) 
following the manufacturer's instructions. Western blots contained $40 \mu \mathrm{g}$ of protein per lane on an $8 \%$ Tris-glycine SDS-polyacrylamide gel electrophoresis. Proteins were transferred to a PVDF membrane (GE, NJ, USA). Blots were blocked in PBS containing 5\% nonfat dry milk powder and incubated overnight at $4{ }^{\circ} \mathrm{C}$ with either anti-mouse $\beta$-catenin mAbs 1:500 dilution ( $\mathrm{R} \& \mathrm{D}$ Systems, MN, USA) or anti-mouse actin mAbs 1:500 dilution (Millipore). Rabbit anti-mouse horse radish peroxidase-linked secondary antibodies $(1: 3,000)$ were used to bind to the primary antibody, and the blot was developed with enhanced chemiluminescence reagent (Pierce, IL) and exposed to film; the image was captured using AlphaImager (CA, USA).

\section{Secondary Sphere Formation}

Primary spheres were obtained by plating 5,000 cells/ $\mathrm{ml}$ in SFM conditions in ultralow adherence culture plates. On day 3, primary spheres were subjected to sensitization to SFRP4 followed by doxorubicin treatment as indicated previously. After treatment, the primary spheres from each condition were disrupted into single cell suspension, and 5,000 cells/ $\mathrm{ml}$ were replated for sphere formation under each condition. On day 3 , the spheres were observed for morphology, total sphere were counted, number of spheres of different sizes were noted using a bright field phase contrast microscope (Nikon- Eclipse TE 2000-S), and photographs were taken using QimagingQICAM-fast 1394. Under 10× magnification, the diameter of each of the spheres was measured using Q-Capture software, and a reference range was set for the different sphere sizes. Large spheres $(7-11 \mu \mathrm{m})$, medium spheres (4-7 $\mu \mathrm{m})$, and small spheres $(<4 \mu \mathrm{m})$ were counted for all drug treatments.

\section{Neuronal Differentiation and Immunofluorescence}

Primary spheres were generated in ultralow adherence plates cultured in SFM. On day 3, primary spheres were subjected to sensitization to sFRP4 followed by doxorubicin treatment as indicated previously. After treatment, the primary spheres from each condition were taken and placed on $1 \%$ gelatin cover slips, and a medium containing $20 \%$ FBS was added for differentiation. Morphological changes were monitored day-wise and photographed. On day 9, the coverslips were subjected to immunohistochemical analysis for differentiation with the neuronal marker $\beta$-III tubulin at 1:1,000 dilution. Nuclei were counterstained with 6-diamidino-2-phenylindole (DAPI; Vector Laboratories) and viewed using a Nikon Eclipse TE2000-U fluorescent microscope, and photographs were taken using QimagingQICAM-fast 1394.

\section{Semiquantitative RT-PCR}

GSCs were subjected to sensitization to sFRP4 followed by doxorubicin treatment as indicated previously, and total RNA was extracted from GSCs after treatment using the RNeasy Plus Mini kit (Qiagen) according to the manufacturer's instructions. A cDNA kit (Invitrogen) was used for RT-PCR. Glyceraldehyde-3-phosphate dehydrogenase (GAPDH) gene was amplified to serve as an internal control. The sequences of the primers, the length of predicted amplified gene fragments, and annealing conditions are shown in Table 1 . The genes were amplified for 35 cycles. Products were separated by $1.5 \%$ agarose gel electrophoresis and detected with ethidium bromide.

\section{Real-Time Quantitative PCR}

The qualitative results of mRNA expression of drugtreated GSCs were further quantified using iQ SYBR Green Supermix (Bio-Rad, Hercules, CA) using a 7500 Real-Time PCR System (Applied Biosystems) according to manufacturer's instructions. The relative abundance of mRNAs was obtained using the comparative cycle threshold method and was normalized to the housekeeping control GAPDH.

\section{Statistical Analysis}

Data represent mean and SE from experiments done in triplicate. A two-sided Student's $t$ test was used to

Table 1. Primers Sequences Used

\begin{tabular}{|c|c|c|c|}
\hline Genes & Primer Sequence & $\begin{array}{c}\text { Annealing } \\
\text { Temperature }\end{array}$ & $\begin{array}{l}\text { Product } \\
\text { Size (bp) }\end{array}$ \\
\hline \multirow[t]{2}{*}{ GAPDH } & Forward CAGAACATCATCCCTGCATCCACT & \multirow[t]{2}{*}{61} & \multirow[t]{2}{*}{185} \\
\hline & Reverse GTTGCTGTTGAAGTCACAGGAGAC & & \\
\hline \multirow[t]{2}{*}{ BAX } & Forward GCTGGACATTGGACTTCCTC & \multirow[t]{2}{*}{61} & \multirow[t]{2}{*}{167} \\
\hline & Reverse TCAGCCCATCTTCTTCCAGA & & \\
\hline \multirow[t]{2}{*}{ CYCLIN D1 } & Forward AACTACCTGGACCGCTTCCT & \multirow[t]{2}{*}{61} & \multirow[t]{2}{*}{165} \\
\hline & Reverse CCACTTGAGCTTGTTCACCA & & \\
\hline \multirow[t]{2}{*}{ CD133 } & Forward TCAGTGAGAAAGTGGCATCG & \multirow[t]{2}{*}{60} & \multirow[t]{2}{*}{313} \\
\hline & Reverse TGTTGTGATGGGCTTGTCAT & & \\
\hline \multirow[t]{2}{*}{$\mathrm{p} 21$} & Forward GAGGCCGGGATGAGTTGGGAGGAG & \multirow[t]{2}{*}{63} & \multirow[t]{2}{*}{220} \\
\hline & Reverse CAGCCGGCGTTTGGAGTGGTAGAA & & \\
\hline
\end{tabular}


determine the significance of any differences. For all statistical analyses, the level of significance was set at a probability of $<0.05$.

\section{RESULTS}

\section{GSC Characterization}

Neurospheres were characterized for the expression of GSC markers CD133 using immunohistochemistry, CD133, nestin, and ABCG2 by RT-PCR (Fig. 1). The GSC-enriched cells were used for further analysis.

sFRP4 and Doxorubicin and Cisplatin Treatment Inhibited Cell Proliferation of GSCs and Non-GSCs

To study the effect of sFRP4 singly and in combination with doxorubicin, different concentrations of sFRP4 (0-200 pg) were tested with $50 \mathrm{ng}$ of doxorubicin on U138MG non-GSCs and GSCs (Fig. 2a, b). A dosedependent inhibition by sFRP4 was seen with maximum inhibition at $125 \mathrm{pg} / \mathrm{ml}(p<0.01, n=3)$ and $50 \mathrm{ng}$ of doxorubicin. Therefore, these concentrations of sFRP4 and doxorubicin were used for all the subsequent drug combination studies. sFRP4 treatment with cisplatin $(5 \mu \mathrm{M})$ also inhibited GSC proliferation (Fig. 2c).

Inhibition of GSCs by SFRP4 and With Doxorubicin and Cisplatin Is Ablolished by the Addition of Wnt3a

To determine if the inhibition by sFRP4 is through the Wnt pathway, Wnt3a was added to the cocktail. We found that SFRP4-mediated inhibition, either singly or in combination with dox or cis, was prevented by Wnt3a (Fig. 2d), thereby suggesting that sFRP4 inhibition is through the Wnt-mediated pathway. Furthermore, the central downstream activator of Wnt signaling, namely $\beta$-catenin, was significantly reduced upon sFRP4 treatment (Fig. 2e), indicating that sFRP4 inhibition functions through the Wnt $\beta$-catenin axis.

\section{Detection of Cell Cycle Arrest and Early Apoptotic Events Observed in sFRP4-Mediated Inhibition}

The BrdU assay (quantifying the cells at the $\mathrm{S}$ phase of cell cycle) for GSCs treated with sFRP4, doxorubicin, sFRP4 and doxorubicin, cisplatin, and sFRP4 and cisplatin showed a decrease in the BrdU incorporation (Fig. 3a). Significant changes $(p<0.05)$ were seen in both the drugs and sFRP4 combinations.

The caspase- 3 activity (an indicator of early apoptosis) of GSCs indicated significant changes $(p<0.01)$ in the sFRP4 and doxorubicin combination treatment in comparison to the untreated cells (Fig. 3b). The JC-1 assay demonstrated a high and statistically significant $(p<0.01)$ mitochondrial depolarization in sFRP4-, doxorubicin-, and combination-treated GSCs (Fig. 3c), indicating earlystage cell death and apoptotic response to the various drug treatments. sFRP4-dox combination-treated GSCs showed the maximum depolarization.
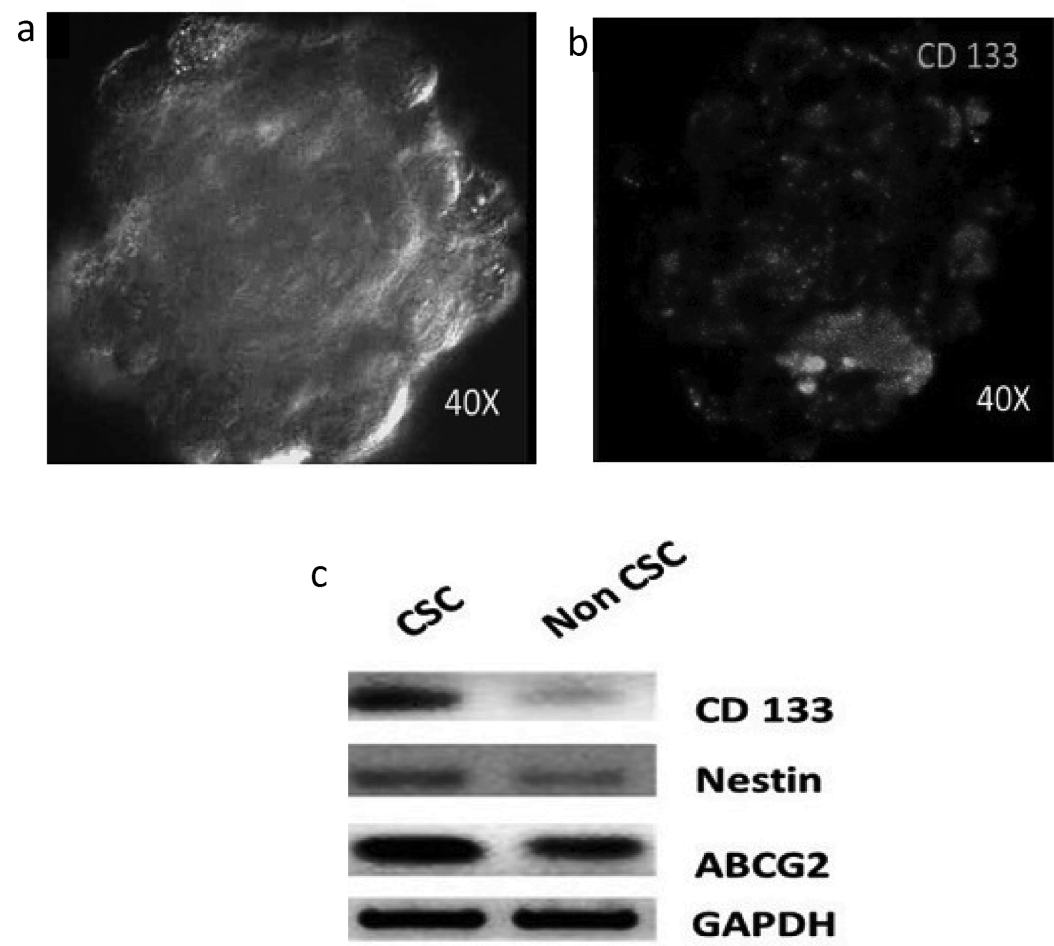

Figure 1. Characterization of GSC population. (a) Neurosphere cultures were maintained in serum-free medium. (b) Neurosphere stained positive for CD133. (c) Increased expression of CD133, nestin, and ABCG2 mRNA was observed in the CSC population. 

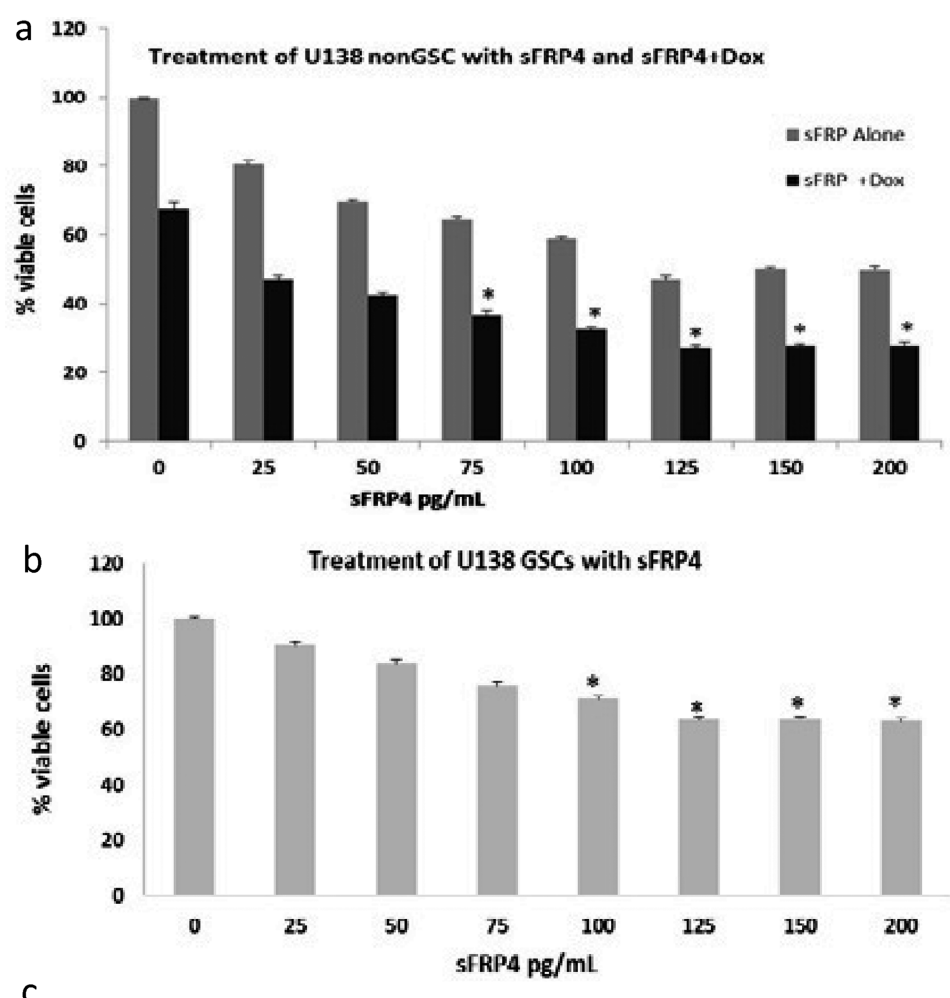

C
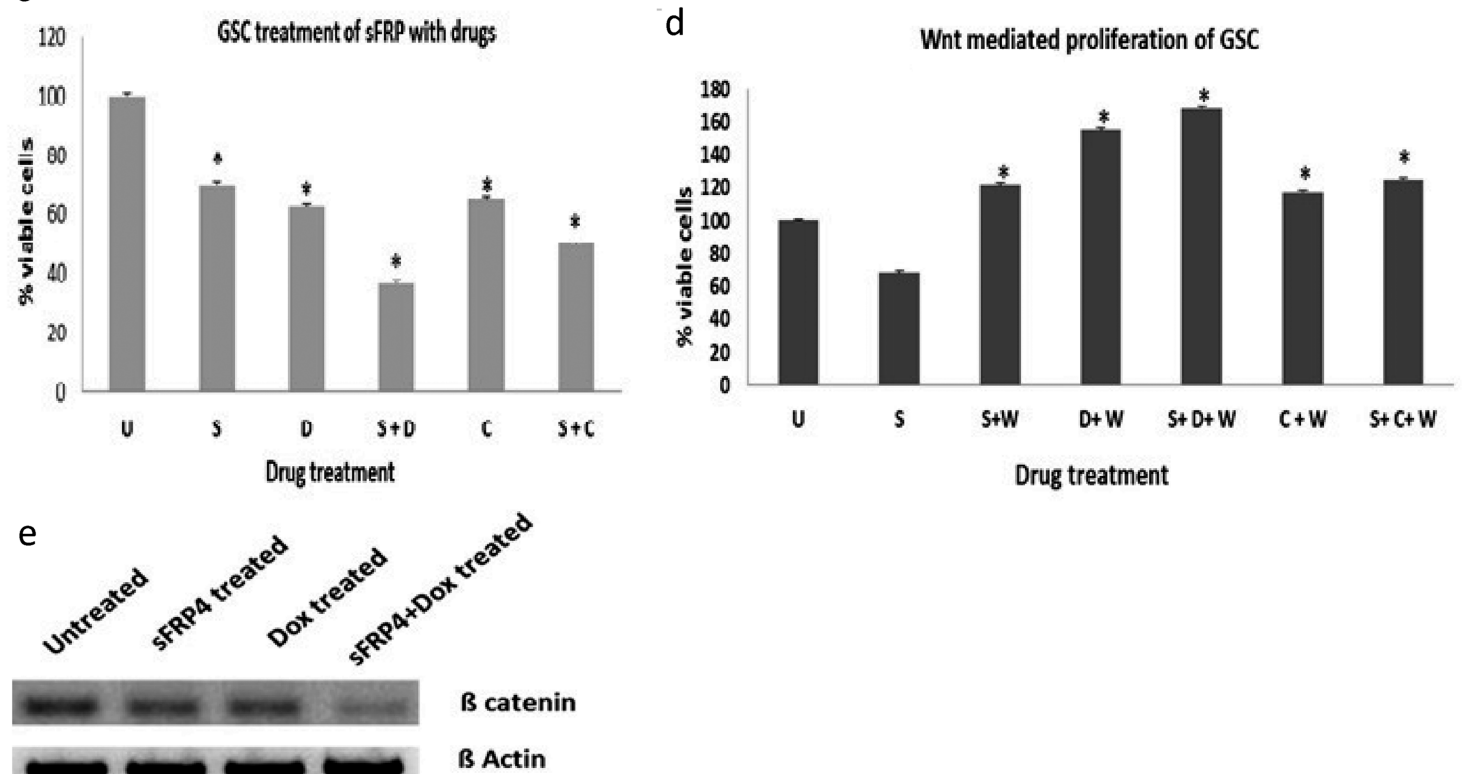

Figure 2. Effect of sFRP4 on U138 non-GSCs and GSCs with drugs. Untreated (U), sFRP4 treated (S), doxorubicin (D), sFRP4 + doxorubicin $(\mathrm{S}+\mathrm{D})$, cisplatin $(\mathrm{C}), \mathrm{sFRP} 4+$ cisplatin $(\mathrm{S}+\mathrm{C}), \mathrm{sFRP} 4+\mathrm{Wnt}(\mathrm{S}+\mathrm{W})$, doxorubicin + Wnt $(\mathrm{D}+\mathrm{W}), \mathrm{sFRP} 4+$ doxorubicin + Wnt $(\mathrm{S}+\mathrm{D}+\mathrm{W})$, cisplatin $+\mathrm{Wnt}(\mathrm{C}+\mathrm{W}), \mathrm{sFRP} 4+$ cisplatin $+\mathrm{Wnt}(\mathrm{S}+\mathrm{C}+\mathrm{W})$. (a) Effect of sFRP4 at various concentrations with doxorubicin $(50 \mathrm{ng} / \mathrm{ml})$ and without doxorubicin on non-GSCs. A dose-dependent inhibition was observed up to $125 \mathrm{pg} / \mathrm{ml}$. (b) Effect of sFRP4 at various concentrations on GSCs. A dose-dependent inhibition was observed up to $125 \mathrm{pg} / \mathrm{ml}$, after which the inhibition was constant. (c) Treatment of GSCs with sFRP4 followed by drugs doxorubicin and cisplatin. Combination treatment with both drugs was more effective than sFRP4 alone or drug alone. (d) Effect of Wnt on sFRP4-mediated inhibition. Inhibition was reversed on the addition of Wnt to both sFRP4 alone and in combination with drugs, thereby showing that inhibition is mediated through the Wnt-Fzd pathway. (e) Expression of $\beta$-catenin protein in drug-treated GSCs. By Western blot analysis, it is seen that $\beta$-catenin decreases significantly upon SFRP4 and doxorubicin treatment and is least expressed in combination treatment. Data are the mean \pm SD of two independent treatments that were performed in triplicate $\left({ }^{*} p<0.01, n=3\right)$. 


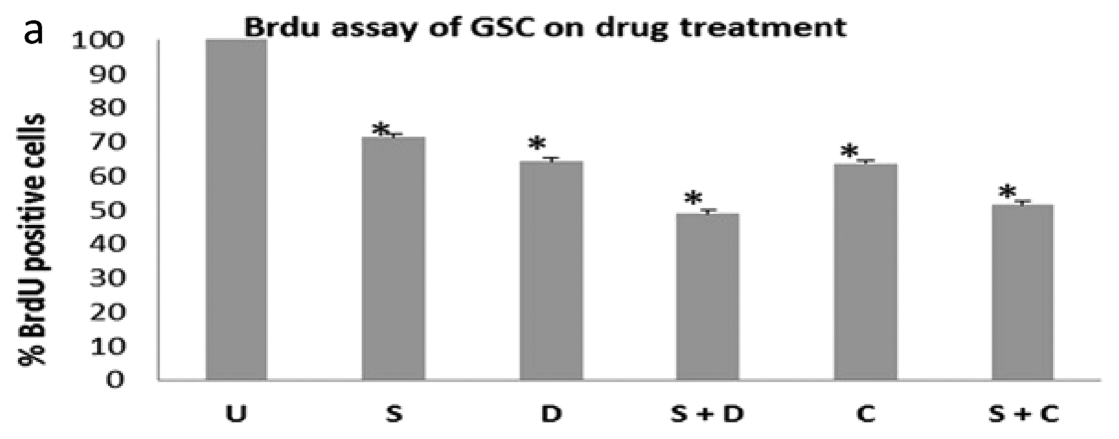

Drug treatment
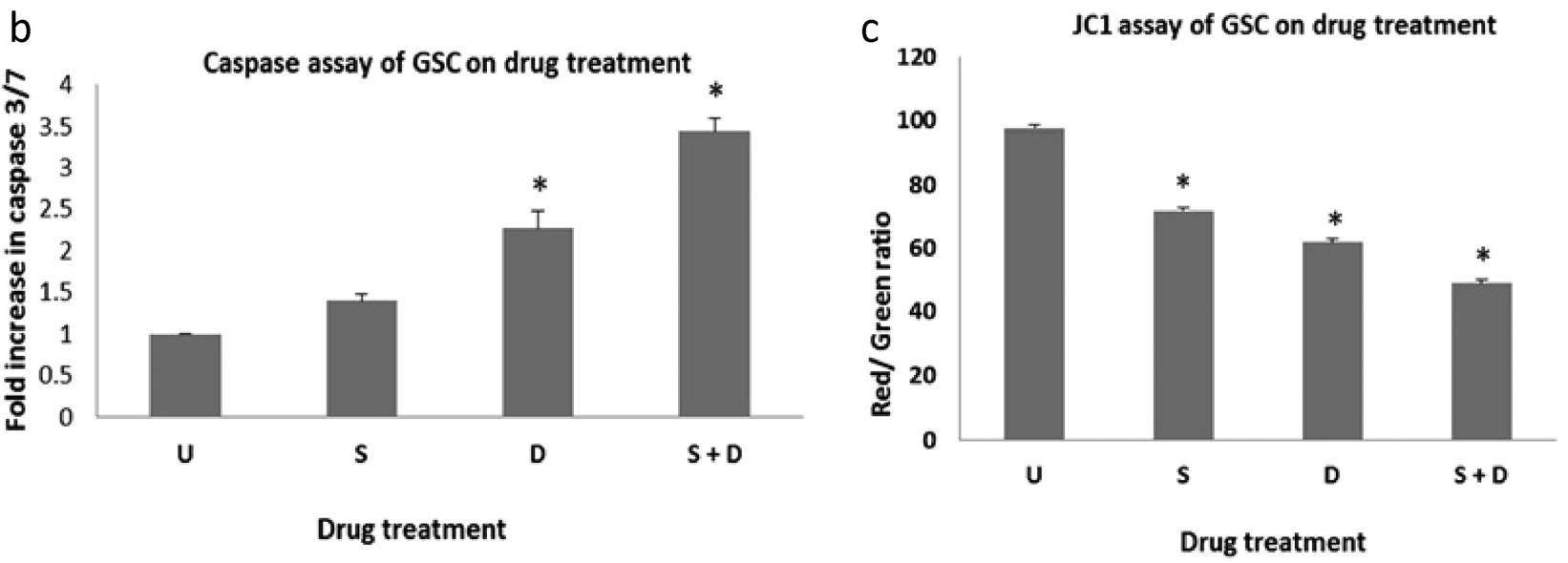

Figure 3. Mechanism of sFRP4 induced cell death on GSCs. Untreated (U), sFRP4 treated (S), doxorubicin (D), sFRP4+doxorubicin $(\mathrm{S}+\mathrm{D})$, cisplatin (C), sFRP4 + cisplatin $(\mathrm{S}+\mathrm{C})$. (a) A BrdU assay to assess GSC proliferation after sensitizing with sFRP4 and subsequent treatment with doxorubicin and cisplatin showed that sFRP4 sensitized GSCs to a higher inhibition with the drugs. (b) Caspase activation by sFRP4 and sFRP4 + doxorubicin-caspase activity was measured in GSCs following drug treatment, and it was seen that apoptosis-mediated caspase-3 activity increased significantly in doxorubicin- and combination-treated GSCs. Data are presented as fold increase in fluorescence as measured relative to untreated cultures. (c) Mitochondrial membrane depolarization studied by JC-1 assay. Mitochondrial membrane potential was measured by JC-1 accumulation in GSCs subjected to sFRP4 and sFRP4 and doxorubicin treatment. Combination-treated cells had the highest loss of membrane potential (lower ratio of red/green indicates loss of potential). Results are the mean $\pm \mathrm{SD}$ of two independent experiments, each performed in triplicate $(* p<0.01, n=3)$.

sFRP4 and Doxorubicin Treatment Reduces the Ability of GSCs to Form Tumor Spheres

Secondary sphere formation of GSCs is indicative of their CSC nature and retention of their stemness. After treatment of GSC spheres with the four-drug combinations, they were analyzed for their ability to form secondary spheres. It was observed that the overall number of spheres formed decreased progressively in the sFRP4-, doxorubicin-, and combination-treated GSCs, with the latter having the least number of spheres (Fig. 4a). Furthermore, the size and quality of the spheres also followed the same pattern as the sphere number, and the largest spheres $(7-11 \mu \mathrm{m})$ were observed mostly only in the untreated and, to a lesser extent, in the sFRP4-treated
GSCs. Conversely, smallest spheres were the most abundant in the combination-treated spheres (Fig. 4a, b).

\section{Proliferation of GSCs Is Reduced and Neuronal Differentiation of GSCs Is Induced by SFRP4 and Doxorubicin Treatment}

To evaluate the differentiation ability of spheres into the neuronal lineage after treatment, the spheres were grown in a differentiation medium for 9 days. On day 2 , the cells started migrating out of the spheres, and differentiation was evident by day 5 . The cells proliferated rapidly out of the spheres in untreated and sFRP4-treated cells, whereas maximum inhibition was seen in combination-treated spheres (Fig. 4c). Differentiation into the 


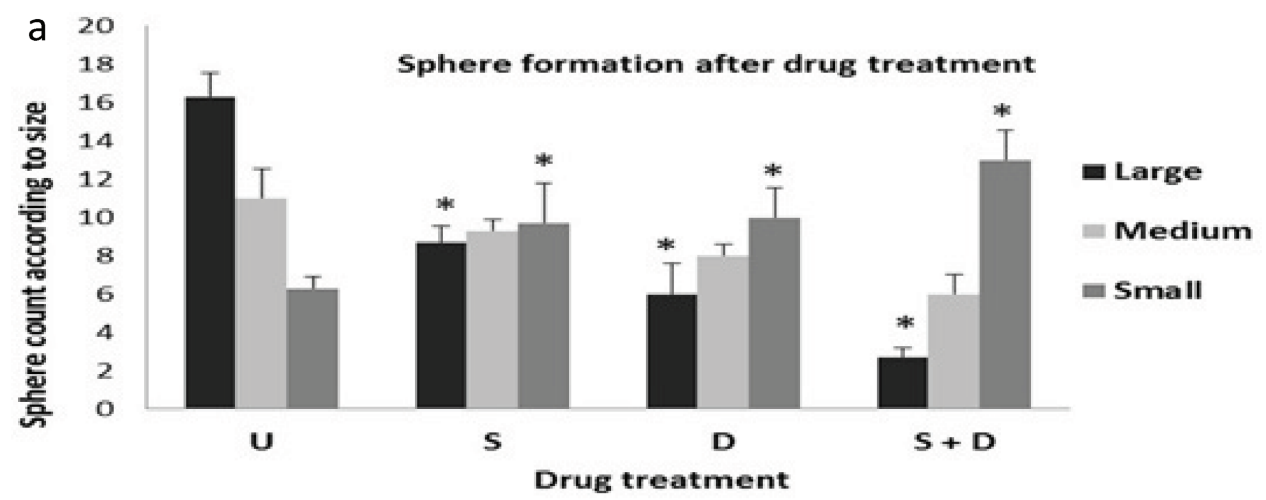

b
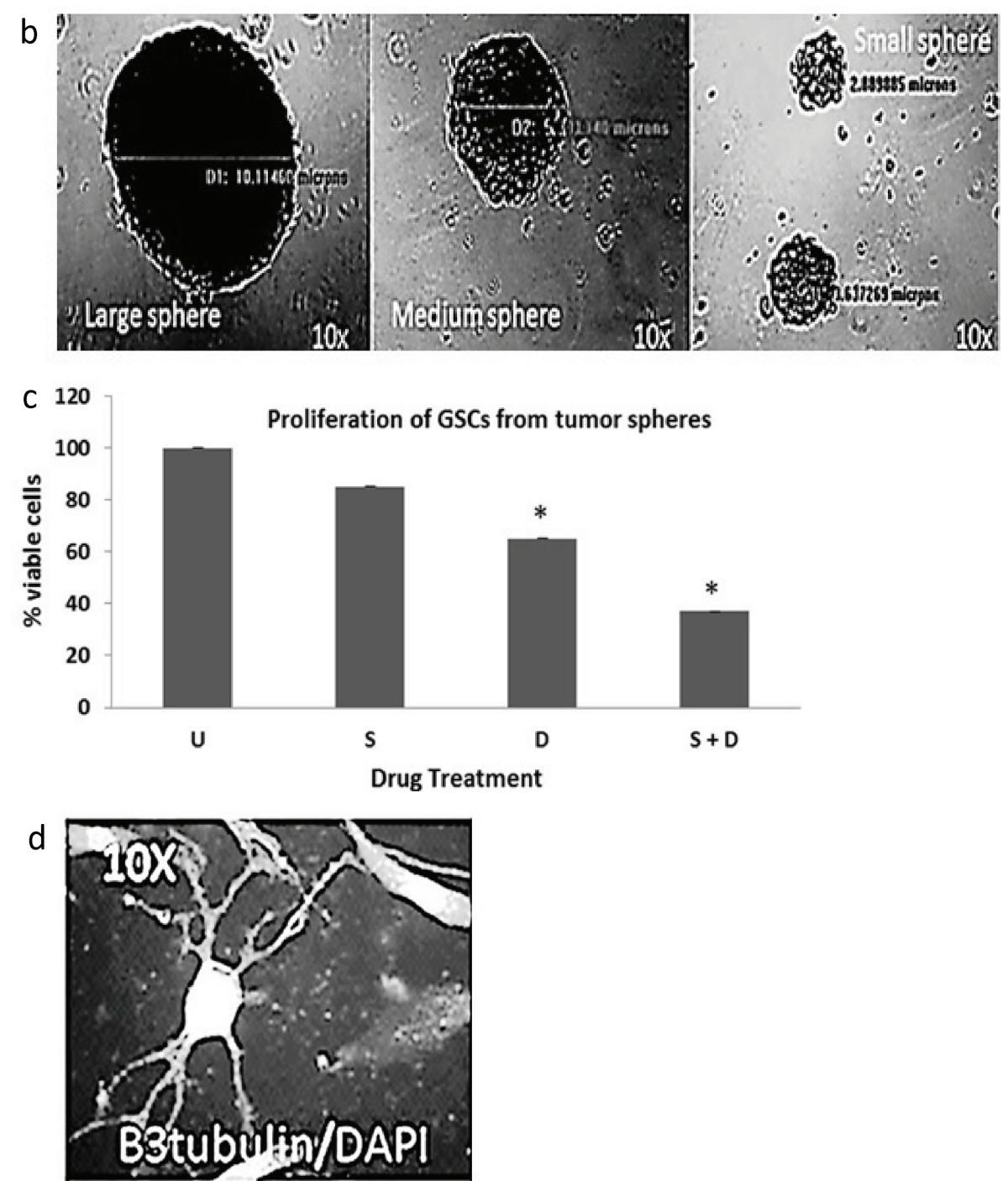

Figure 4. sFRP4 treatment with drug reduces tumorigenicity and induces neuronal differentiation. Untreated (U), sFRP4 treated (S), dox (D), sFRP4 + dox (S+D). (a) Sphere formation was measured for size (large, medium, and small) as shown in (b) and number after drug treatment (higher number of large spheres indicates viable GSCs). Results are the mean \pm SD of two independent experiments and in triplicates each $(* p<0.01, n=3)$. (c) Spheres cultured in differentiation medium after drug treatment. Untreated spheres proliferated rapidly out of spheres, whereas combination-treated spheres had the slowest rate of proliferation. Results are the mean \pm SD of two independent experiments and in triplicates each $(* p<0.01, n=3)$. (d) Immunohistochemistry for detecting neuronal differentiation. Using the neuronal specific antibody $\beta$-III-tubulin, the neuronal structures observed in the combination-treated spheres were positive for this marker and showed a characteristic morphology with axonal and dendritic processes. A secondary control (first panel) does not show nonspecific labeling of the cells. 
neuronal lineage, using the neuronal marker $\beta$-III tubulin, was observed after the combination treatment (Fig. 4d).

\section{Apoptotic Genes Differentially Expressed and GSC Marker CD133 Decreased by SFRP4 and Doxorubicin Treatment}

Analysis of proapoptosis gene transcripts (Bax, p21), $\beta$-catenin-regulated and pro-proliferation gene $(\mathrm{CycD} 1)$, and the glioma stem cell marker (CD133) was done by semiquantitative PCR to obtain a trend (Fig. 5a) and further quantified by quantitative PCR (Fig. 5b-e). Bax induces apoptosis with an early release of cytochrome $\mathrm{C}$ preceding many apoptosis-associated morphological alterations and caspase activation and subsequent substrate proteolysis (6). It was observed that Bax clearly increased with very low expression in the untreated groups and an increase in a stepwise manner with sFRP4, doxorubicin, and combination treatment, with the latter having the highest expression levels (Fig 5b). Expression of p21 increased in all drug treatments (Fig. 5c). Expression of the $\beta$-catenin-regulated oncogene CycD1 decreased in sFRP4-treated, doxorubicin-treated, and combination-treated GSCs compared to the control, showing about a threefold decrease in treated samples (Fig. 5d). Expression of the glioma stem cell marker CD133 was analyzed to determine whether the tumorinitiating population was decreased upon drug treatment.
sFRP4 treatment drastically reduced CD133 expression, and combination treatment almost completely abolished its expression (Fig. 5e). This suggests that the sFRP4 treatment is effective against the CSCs too.

\section{DISCUSSION}

In this study, we show that sFRP4, by itself and in combination with chemotherapeutics, elicits antiproliferative activity against glioma cells and GSCs. CSCs, which are analogous to normal stem cells, are a small population of cells at the origin of the tumor and are responsible for tumor growth and maintenance (7). We have recently identified CSCs from the glioma cell line U138MG, based on their ability to form neurospheres in nonadherent, serumfree conditions, high expression of CSC markers CD133 and nestin, and a high degree of chemoresistance (5). The drug-resistant nature of GSCs is partly rendered by the high expression of drug effluxing proteins, as reported in U138MG as well as other glioma cell lines (8).

The sFRP genes function as tumor suppressors, being transcriptionally silenced by DNMT-mediated demethylation (9). sFRP4 has been shown to have antiproliferative and proapoptotic roles during normal homeostasis in tissues such as ovary, corpus luteum, placenta, and mammary gland (10-12). Apoptosis was induced and proliferation was suppressed when sFRP4 expression was restored in mesothelioma cell lines (13). Conversely, in mesothelioma,
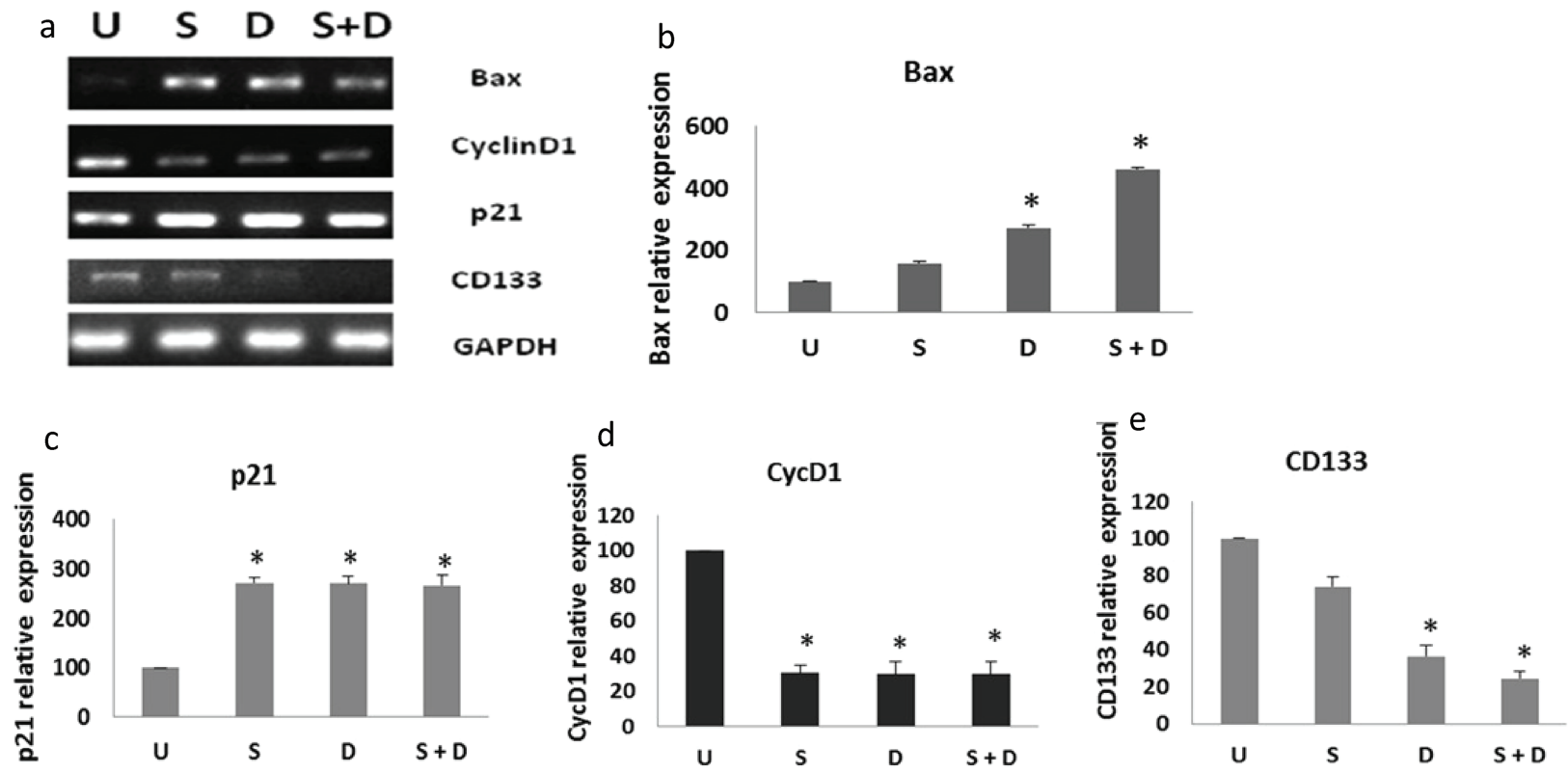

Figure 5. Effect of sFRP4 on the expression of apoptotic and stemness genes. mRNA expression of Bax (b), p21 (c), CycD1 (d), CD133 (e) after sFRP4, doxorubicin, and combination treatment by semiquantititative PCR (a) and qPCR (b-e). (a) Total RNA isolated from cells was analyzed by semiquantitative PCR showing untreated (U), sFRP4 treated (S), doxorubicin (D), sFRP4+doxorubicin $(\mathrm{S}+\mathrm{D})$. (b, c) Bax and p21 increased in all treatments, with Bax showing highest expression in the sFRP+doxorubicin-treated GSCs. (d, e) CycD1 and CD133 decreased in all treatments, with CD133 showing lowest expression in the sFRP+doxorubicin-treated GSCs. Gene expression is represented as relative mRNA levels following normalization by housekeeping gene expression. Results are mean \pm SD for three cultures $(* p<0.01, n=3)$. 
inhibition of drug-induced apoptosis resulted when SFRP4 was suppressed (14). Inhibition of U138MG by sFRP4, as demonstrated in our study, further proves that sFRP4 is able to induce apoptosis. Reduction in GSC number and neurosphere formation further confirmed that sFRP4 was effective in suppressing both the tumor parenchymal cells and stem-like cells. Naturally occurring Wnt antagonists, such as Dkk, contribute to chemosensitizing tumors to therapeutics such as cisplatin in head and neck cancers (15). In this study, we observed a distinct increased response to drugs after both monolayer cells and GSCs were initially sensitized with sFRP4. This inhibition was reversed by Wnt3a, and upon sFRP4 treatment there was a subsequent decrease in $\beta$-catenin expression. We can thus infer that Wnt $\beta$-catenin signaling plays an important role in the acquisition and modulation of chemoresistance in glioma tumors and GSCs. This is the first report that demonstrates sFRP4 can sensitize GSC-enriched cells to drugs such as doxorubicin and cisplatin.

Molecular analysis of apoptosis in both normal tissue and tumors has established a positive relationship between increased expression of sFRP4 and apoptosis $(16,17)$. Not many pathways have been identified so far that are functional in GSCs' resistance to apoptotic signals. We observed that the inhibition of GSC-enriched cells induced by sFRP4 and its chemosensitization to doxorubicin and cisplatin is mediated through the apoptotic pathway. This was shown by an increase in caspase-3, which plays a central role in the execution of cell apoptosis (18), overexpression of proapoptotic genes such as Bax, and reduced expression of the prosurvival gene cyclin D1. We observed a gradual increase in Bax expression on drug treatment, which peaked in SFRP4- and doxorubicintreated GSCs, showing that the priming by sFRP4 and subsequent treatment with doxorubicin activated this proapoptotic protein more than treatment with only sFRP4 or doxorubicin. In the case of CycD1 expression, all three drug treatments had a similar decrease when compared to the untreated group, suggesting that the relationship between CycD1 inhibition and Bax expression is not inversely proportional. To establish a link between Bax and p53-mediated apoptosis, we looked into the expression of $\mathrm{p} 21$ following drug treatment. A key downstream target of p53, the cyclin-dependent kinase (cdk) inhibitor p21 mediates both $\mathrm{G}_{1}$ and $\mathrm{G}_{2} / \mathrm{M}$ phase arrest (19). The modest increase in expression of $\mathrm{p} 21$ in the entire drugtreated GSCs in our study indicated the role of the tumor suppressor p53 in apoptotic stress.

The reduction in proliferation of GSCs that we observed in this study, by the BrdU, caspase-3, and JC-1 assays, indicated that SFRP4 induced chemosensitization by inducing the apoptotic machinery. sFRP4 sensitization at low concentrations most likely primes proapoptotic gene expression through $\beta$-catenin-dependent pathways.
In our study, we observed that Wnt3a activates proliferation of sFRP4-, doxorubicin-, cisplatin-, and drug combination-treated GSCs. This could also be due to a hyperproliferation of the resistant cell population in addition to the inhibition of the drug. Wnt3a increases the level and nuclear translocation of FoxM1, which binds to $\beta$-catenin and promotes $\beta$-catenin nuclear localization and transcriptional activity (20). A similar mechanism may be operational in GSCs too, which can only be confirmed by studying the induction of FoxM1 and subsequent nuclear accumulation of $\beta$-catenin. We were able to show that sFRP4 and doxorubicin combination treatment decreased sphere formation, thereby suggesting a reduction in the GSC population. Further evidence for a decrease in stemness of the GSCs was provided in the differentiation assays that showed that the combination treatment of sFRP4 and doxorubicin not only decreased proliferation from the GSC spheres but also induced neuronal differentiation. Campos et al. (21) induced differentiation of GSCs with all-trans retinoic acid and found an increase in antimigratory, antiangiogenic, and chemosensitizing effects in vitro and decreased the tumor-initiating capacity of these cells in vivo. In our study, another evidence for the reduction of GSCs was provided by a drastic decrease in the CD133 marker, especially in cells that were sensitized to sFRP4 before doxorubicin treatment. In vivo tumorigenicity assays in immunocompromised mice after drug treatment will further confirm the decrease in the tumor-initiating cell population.

To summarize, sFRP4 chemosensitized glioma cells so that the pro-oncogenic profile was reduced, neuronal differentiation was initiated, and cancer stem cell traits were decreased, which strengthens the therapeutic significance of this protein in the treatment of gliomas. Additional studies from patient-derived cancer stem cell line and in an in vivo brain tumor mouse model would confirm whether the sFRP4 by itself and with functional chemotherapeutics would abolish the CSC population and effectively reduce the tumor load to prevent the recurrence and relapse of the deadly glioma.

ACKNOWLEDGMENTS: We would like to thank Mr. Greg Cozens and Dr. Frank Arfuso, School of Anatomy, Physiology and Human Biology, the University of Western Australia, Perth, Australia, and Dr. Simon Fox, School of Pharmacy, Curtin University, Perth, Australia, for providing primers for real-time PCR. This work was supported by the India Initiative Funds of the University of Western Australia provided by Prof. Arun Dharmarajan and Prof. Michael Millward.

\section{REFERENCES}

1. Zhuand, J.; Parada, L. F. The molecular and genetic basis of neurological tumours. Nat. Rev. Cancer 2:616-626; 2002.

2. Islam, M. O.; Kanemura, Y.; Tajria, J.; Mori, H.; Kobayashi, S.; Shofuda, T.; Miyake, J.; Hara, M.; Yamasaki, M.; Okano, H. Characterization of $\mathrm{ABC}$ transporter $\mathrm{ABCB} 1$ 
expressed in human neural stem/progenitor cells. FEBS Lett. 579:3473-3480; 2005.

3. Kawano, Y.; Kypta, R. Secreted antagonists of the Wnt signalling pathway. J. Cell Sci. 116:2627-2634; 2003.

4. Saran, U.; Arfuso, F.; Zeps, N.; Dharmarajan, A. Secreted frizzled-related protein 4 levels reflect the responsiveness of ovarian cancer cell lines in vitro and are associated with tumour grade in vivo. BMC Cell Biol. 2121:13-25; 2012.

5. Warrier, S.; Pavanram, P.; Raina, D.; Arvind, M. Study of chemoresistant CD133+ Cancer Stem Cells from human glioblastoma cell line U138MG using multiple assays. Cell Biol. Int. 36:1137-1143; 2012.

6. Beltran, E.; Fresquet, V.; Martinez-Useros, J.; RichterLarrea J. A.; Sagardoy, A.; Sesma, I.; Almada, L. L.; Montes-Moreno, S.; Siebert, R.; Gesk, S; Calasanz, M. J.; Malumbres, R.; Rieger, M; Prosper, F.; Lossos, I. S.; Piris, M. A.; Fernandez-Zapico, M. E.; Martinez-Climent, J. A. A cyclin-D1 interaction with BAX underlies its oncogenic role and potential as a therapeutic target in mantle cell lymphoma. Proc. Natl. Acad. Sci. U. S. A. 26:12461-12466; 2011.

7. Lobo, N. A.; Shimono, Y.; Qian, D.; Clarke, M. F. The biology of cancer stem cells. Annu. Rev. Cell Dev. Biol. 23:675-699; 2007.

8. Bleau, A. M.; Hambardzumyan, D.; Ozawa, T.; Fomchenko, E. I.; Huse J. T.; Brennan, C. W.; Holland, E. C. PTEN/PI3K/ Akt pathway regulates the side population phenotype and ABCG2 activity in glioma tumor stem-like cells. Cell Stem Cell 4:226-235; 2009.

9. Linhart, H. G.; Lin, H.; Yamada, Y.; Moran, E.; Steine, E. J.; Gokhale, S.; Lo, G.; Cantu, E.; Ehrich, M.; He, T.; Meissner, A.; Jaenisch, R. Dnmt3b promotes tumorigenesis in vivo by gene-specific de novo methylation and transcriptional silencing. Genes Dev. 21:3110-3122; 2007.

10. Hsieh, M.; Mulders, S. M.; Friis, R. R.; Dharmarajan, A.; Richards, J. S. Expression and localization of secreted frizzled-related protein-4 in the rodent ovary: Evidence for selective up-regulation in luteinized granulosa cells. Endocrinology 144:4597-4606; 2003.

11. Lacher, M. D.; Siegenthaler, A.; Jäger, R.; Yan, X.; Hett, S.; Xuan, L.; Saurer, S.; Lareu, R. R.; Dharmarajan, A. M.; Friis, R. Role of DDC-4/sFRP-4, a secreted frizzled-related protein, at the onset of apoptosis in mammary involution. Cell Death Diff. 10:528-538; 2003.

12. Constantinou, T.; Baumann, F.; Lacher, M. D.; Saurer, S.; Friis, R.; Dharmarajan, A. SFRP-4 abrogates Wnt-3ainduced beta-catenin and Akt/PKB signalling and reverses a Wnt-3a-imposed inhibition of in vitro mammary differentiation. J. Mol. Signal. 2:3-10; 2008.

13. He, B.; Lee, A. Y.; Dadfarmay, S.; You, L.; Xu, Z.; Reguart, N.; Mazieres, J.; Mikami, I.; McCormick, F.; Jablons, D. M. Secreted frizzled-related protein 4 is silenced by hypermethylation and induces apoptosis in beta-catenindeficient human mesothelioma cells. Cancer Res. 65:743$748 ; 2005$.

14. Lee, A. Y.; He, B.; You, L.; Dadfarmay, S.; Xu, Z.; Mazieres, J.; Mikami, I.; McCormick F.; Jablons, D. M. Expression of the secreted frizzled-related protein gene family is downregulated in human mesothelioma. Oncogene 23:6672-6676; 2004.

15. Gosepath, E. M.; Eckstein, N.; Hamacher, A.; Servan, K.; von Jonquieres, G.; Lage, H.; Györffy, B.; Royer, H. D.; Kassack, M. U. Acquired cisplatin resistance in the headneck cancer cell line Cal27 is associated with decreased DKK1 expression and can partially be reversed by overexpression of DKK1. Int. J. Cancer 123:2013-2019; 2008.

16. Agarwal, R.; Kaye, S. B. Ovarian cancer: Strategies for overcoming resistance to chemotherapy. Nat. Rev. Cancer 3:502-516; 2003.

17. Balch, C.; Huang, T. H.; Brown, R; Nephew, K. P. The epigenetics of ovarian cancer drug resistance and resensitization. Am. J. Obstet. Gynecol. 191:1552-1572; 2004.

18. Liu, X.; Kim, C. N.; Yang, J.; Jemmerson, R.; Wang, X. Induction of apoptotic program in cell-free extracts: Requirement for dATP and cytochrome c. Cell 86:147-157; 1996.

19. Ando, T.; Kawabe, T.; Ohara, H.; Ducommun, B.; Itoh, M.; Okamoto, T. Involvement of the interaction between $\mathrm{p} 21$ and PCNA for the maintenance of G2/M arrest after DNA damage. J. Biol. Chem. 16; 276:42971-42977; 2001.

20. Zhang, N.; Wei, P.; Gong, A.; Chiu, W. T.; Lee, H. T.; Colman, H.; Huang, H.; Xue, J.; Liu, M.; Wang, Y.; Sawaya, R.; Xie, K.; Yung, W. K.; Medema, R. H.; He, X.; Huang, $\mathrm{S}$. FoxM1 promotes $\beta$-catenin nuclear localization and controls Wnt target-gene expression and glioma tumorigenesis, Cancer Cell 20:427-442; 2011.

21. Campos, B.; Wan, F.; Farhadi, M.; Ernst, A.; Zeppernick F.; Tagscherer K. E.; Ahmadi, R.; Lohr, J.; Dictus, C.; Gdynia, G.; Combs, S. E.; Goidts, V.; Helmke, B. M.; Eckstein, V.; Roth, W.; Beckhove, P.; Lichter, P.; Unterberg, A.; Radlwimmer, B.; Herold-Mende, C. Differentiation therapy exerts antitumor effects on stem-like glioma cells. Clin. Cancer Res. 16:2715-2728; 2010. 